$\xi=$

\title{
IOT Based Monitoring Of Household Electricity Appliances
}

\author{
K M. Sri Lakshmi ${ }^{1}$, P. Sairam ${ }^{2}$, V. Yeswanth ${ }^{3}$, A. Akhila ${ }^{4}$ \\ ${ }^{1,2,3,4}$ Department of CSE, Koneru Lakshmaiah Education Foundation, Vaddeswaram, Guntur,Andhra Pradesh,India-522502 \\ *Corresponding author E-mail: manchala.srilakshmi@kluniversity.in
}

\begin{abstract}
A Smart Electricity meter is a new kind of electricity meter that can send meter reading and billing to the user. They are a replacement for standard meters, which use technology created decades ago .Smart electricity meters have been in development in many countries. Presently, people have to come to electricity meter to generate bill. We could not know how many units of current we have been consuming from time to time. The system we propose in this paper is a smart electricity meter which will monitor the current electricity usage and gives a notification to us which says how many units of current have been consumed and the amount we have to pay. we can also enable a setting that will notify us whenever particular units of current have been consumed. The entire consumption history of current from time to time can be monitored in the database.
\end{abstract}

Keywords: Database, Internet of Things, Smart Electricity meter

\section{Introduction}

The smart power meter which is mounted in the home/office records the consumption of electric energy from time to time and shows it on either a number dial or advanced show. When the billing cycle ends an individual from the organization needs to visit the meter and record the reading for additionally charge age. The present smart power charging/billing scheme is error-prone and monotonous[1]. The main agenda of any innovation is to relieve human efforts, having said that, irrespective of the hurdles we embarked on such a goal. In straightforward terms, a smart power meter can be described as an instrument which measures the power consumed by a living arrangement, business, houses, or electrically fueled gadgets or machines.

The power meters measure the power usage in terms of units, and the most widely accepted one is the kilowatt-hour [kWh]. Sporadic readings of power meters set up billing cycles and power consumed during the cycle, the cycle most of the time turns out to be a month. [2][3]. The Microcontroller framework perpetually collects all the consumption data and the client can be provided with the real-time readings after the power office individual approves it. The SMS helps in projecting a wide variety of administrations to cell phone clients. SMS comes in handy to send an update or note especially when the client is busy and not able to react abruptly.

This kind of Energy Monitoring System which we are developing is suitable in any case where an electrical framework is employed like industries, business buildings, etc. This framework provides benefits to both power office as well as clients: efficient power tracking and control to power office, on the other hand, easy bill installment for users. Our device aids in not only delivering the electricity bill but also in saving time by making the payments easier, as time is valuable and it plays a key role in our lives.

\section{Brief over View of the Project}

In this design we give an exceptional registered number for each smart power meter. This registered number is coupled to SIM card exceptional organization number. This structure constantly screens power meter, SMS is sent to the association according to user need. We can select the time in the microcontroller to each meter's reading step by step, step by step, month to month and sends it to central server of the smart power Supplier organization. Then the message is sent to 3 showed mobile numbers saved in the microcontroller.

The meter's reading is secured in database by SMS door .charge is sent to the end user by figuring out the perusing, which is set in database. Bill generated from smart power Supplier corporation will be sent by SMS. Then again we can similarly send through either by web account, by post and by email. This system offers versatility to the customer for paying the bill.

AMR also sends the information of the power cut, control load, and smart power provider can in like manner cut the affiliation if customer does not require the relationship through SMS inquire. This SMS card advantage number is used to recognize and recuperate customers detail for charging and ID reason.

\section{GSM Based Energy Meter Billing Via SMS}






Figure 1: Schamtic layout

\section{Description of Wireless Energy Meter}

This fig shows piece layout of our structure. In this structure steady checking and recording the data of smart power meter is done. This is refined by techniques for a scaled down scale controller (89S52). We will have an interface with LCD near the customer for convince which demonstrates the power used and cost until that period... Scaled down scale controller unit interminably screen the smart power meter besides, beats appear on LCD. For the information of control crossbreed microcontroller unit is interface with RTC clock and hand-off and for correspondence it also interfaced with GSM modem by using MAX232. We have picked the AT89S52 little scale controller in view of its low power, world class CMOS. It is a 8-bit littler scale controller with $8 \mathrm{~K}$ bytes of in-structure programmable flicker memory. It furthermore gives 256 bytes on chip RAM, 32 I/O lines, Watch puppy clock, two data pointers, three 16 bit clock/counters a full duplex serial port, on-chip oscillator and clock equipment.

\section{Technical Standard Standards Required for the Proposed Design}

The subtle elements used for the arrangement and progression of this remote power/energy meter are given underneath. This system is also used to separate the control supply to the house if there ought to be an event of default of charge and besides if there ought to be an event of considerable usage of load than decided.

1. Input Supply voltage: $230 \mathrm{~V}$

2. Frequency range for the operation of the device: $50 \mathrm{HZ}$

3. Assigned GSM modem: Tri band GSM modem

4. MC input supply voltage: $5 \mathrm{~V}$

5. Display type: LCD display

\section{Implementation and Result}

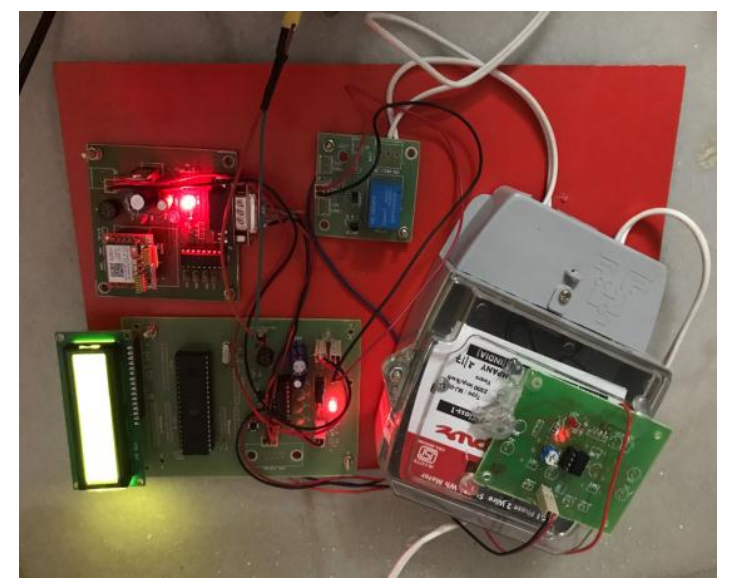

Figure 1: - Experimental setup for proposed design

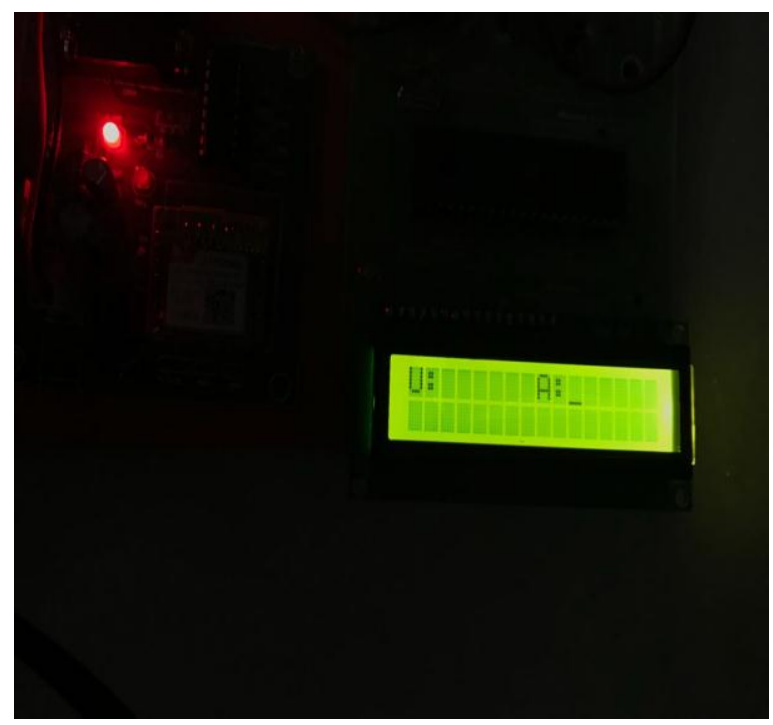

Figure 2 :- Indicates U:Units and A:Amount

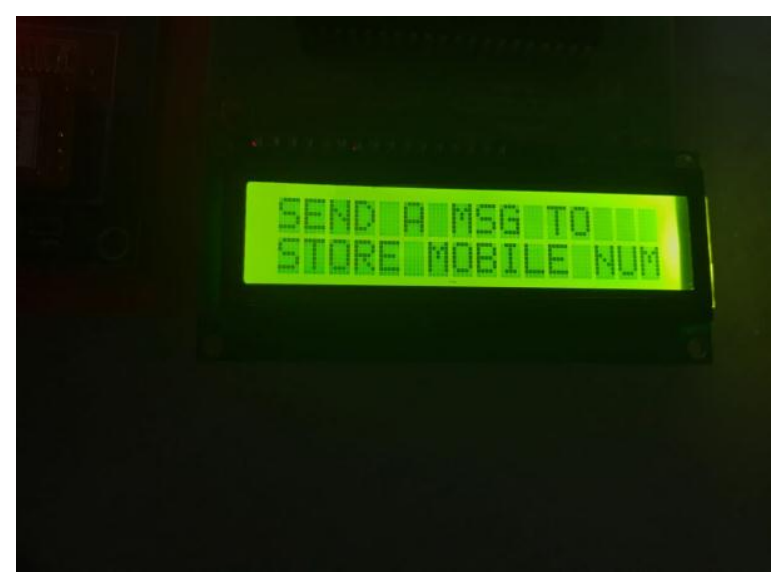

Figure 3:- Send a message to Registered Mobile 


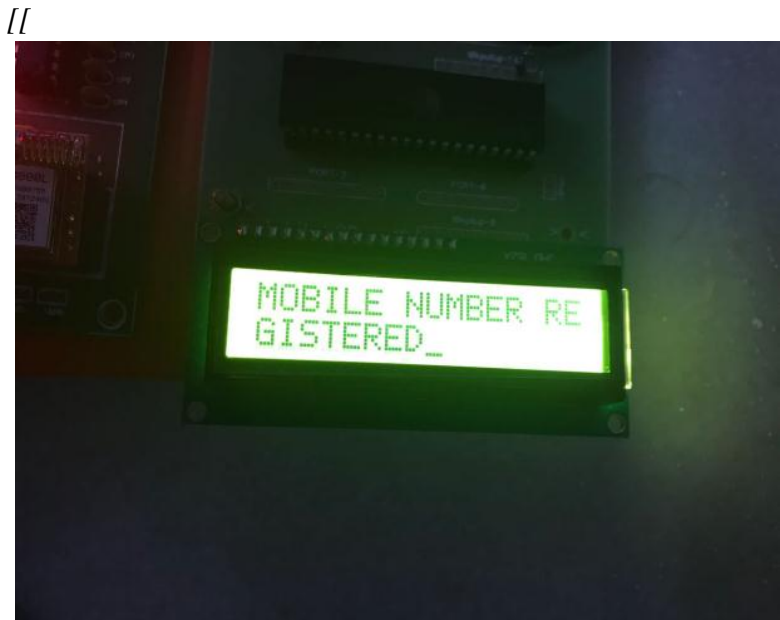

Figure 4:-Mobile number Registered

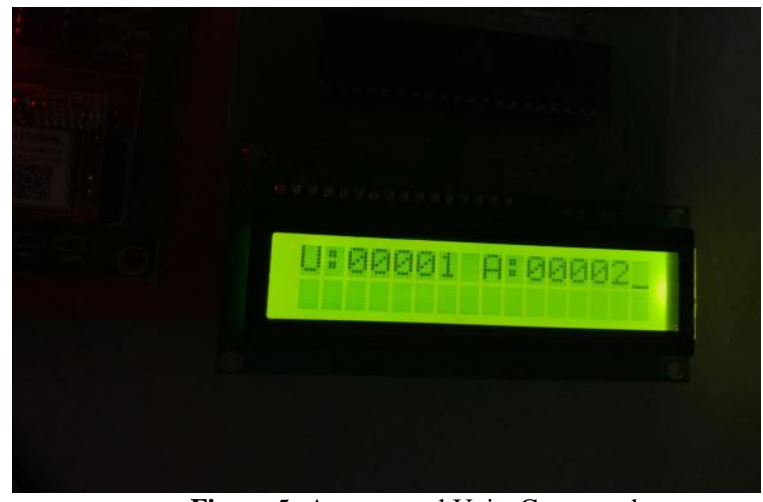

Figure 5:-Amount and Units Consumed

This is our structure were smart power meter is related with the little scale controller one side and afterward again it is related with the essential supply. Here in the controller we form a code for recuperating the data from the smart power meter. From little scale controller we interface it to ADC which changes over basic data to the propelled data and negative behavior pattern versa from here the data is given to the MAX 232 and RS232 which are used as interfacing unit between smart power meter and the GSM network [6]. RS232 is a connector which is used to transmit the electric signs between the system and modem. As per the code written in the littler scale controller, SMS is sent to the smart power Provider Company. At whatever point SMS is sent to the smart power Provider Company then customer is given a prepared alert which moreover reminds customer about the charge. This is for the customer flexibility here we too give LCD Display. This helps the customer for affirming the data when SMS is sent to the smart power Supplier Company. This decreases bungles done by the masters in the midst of taking the smart power meter perusing.

\section{Working of Project}

The smart power meter records the measure of smart power usage. It does all things considered by an electromechanical system. The structure is outfitted with such a framework, to the point that an expansion in measure of current travel through circuit influences the hover to turn speedier, suggests that the rotational speed of plate is particularly comparing to the measure of current traveling through circuit. This insurgency effect of circle influences the apparatus to instrument work in like way and in that amount shape rate of smart power usage grows the gleaming rate of LED consolidated inside the meter. The beats from this LED are supported to microcontroller for check operation i.e. these beats are checked by microcontroller and readings are secured into outside memory.
Outside memory used here, is EEPROM .This memory can store past database additionally if one needs to check past usage status.LCD is related with microcontroller keeping in mind the end goal to exhibit the present status of GSM Modem.GSM modem is the best approach to give over remote systems.GSM modem is related with microcontroller by methods for MAX 232 IC.GSM modem passes on at RS232 standard voltage levels while uC appreciates TTL reason levels so MAX 232 fills in as voltage level converter. It changes over Rs 232 levels into TTL and the different way. At whatever point a charge is sent to GSM modem, it deciphers that request and works suitably.

\section{Conclusion}

The work that we have proposed sees that people using these meters do not face any kind of problems with controlling their household appliances. And also see that people could control their usage of electricity. So that people can get their update of electricity usage from time to time. The work we propose can be used to preserve electricity for the future people can control their electrical appliances from any where they want to control it and it will help them reduce unnecessary use of electricity.

\section{Future Scope}

In future we would like see make other kind of changes so that it will be help full in decreasing the manufacture cost. Also see that working of this meter is made much simpler for the use of elder people and less educated people. We will also see that there are no issues with the validity the meter and security should be well developed that other people can't easily access the database or the billing system and make any kind of changes to bill generated by the system.

\section{References}

[1] YujunBao and Xiaoyan Jiang, Outline of electric Energy Meter for long-remove information data exchanges which in light of GPRS, ISA 2009. Worldwide Workshop on Intelligent Systems and Applications, 2009.

[2] .H.G.RodneyTan,C.H.Lee,V.H.Mok,Automatic control meter perusing framework utilizing GSM organize, The eighth International Power Engineering Conference (IPEC 2007).

[3] Vivek Kumar Sehgal,Nitesh Panda, NipunRaiHanda, Electronic Energy Meter with moment billing,UKSim Fourth European Modeling Symposium on Computer Modeling and Simulation.

[4] Bharath P, Ananth N, Vijetha S, JyothiPrakash K. V., Remote mechanized computerized Energy Meter, ICSET 2008.

[5] P.K. Lee and L.L. Lai, Fieee, A commonsense way to deal with remote GPRS on-line control quality checking framework, Power Engineering Society General Meeting, 2007.

[6] SubhashisMaitra, Installed Energy Meter another idea to quantify the vitality devoured by a customer and to pay the bill, Power System Technology and IEEE Power India Conference, 2008.

[7] T El-Djazairy, B J Beggs and I F Stewart, Investigation of the utilization of the Global System for Mobile Communications (GSM) arrange for metering and load administration telemetry, Electricity Distribution. Section 1: Contributions. CIRED.14th International Conference and Exhibition on (IEE Conf. Publ. No. 438). 